\title{
Role of the phase in the identification of delay time in semiconductor lasers with optical feedback
}

\author{
Romain Modeste Nguimdo,* Miguel C. Soriano, and Pere Colet \\ Instituto de Física Interdisciplinar y Sistemas Complejos, IFISC (CSIC-UIB), Campus Universitat de les Illes Balears, \\ E-07122 Palma de Mallorca, Spain \\ ${ }^{*}$ Corresponding author: modeste@ifisc.uib-csic.es
}

Received August 1, 2011; revised October 6, 2011; accepted October 6, 2011; posted October 10, 2011 (Doc. ID 152053); published November 9, 2011

We consider a semiconductor laser with external optical feedback operating at a regime for which the delay time signature is extremely difficult to identify from the analysis of the intensity time series, using standard techniques. We show that such a delay signature can be successfully retrieved by computing the same quantifiers from the phase, the real or the imaginary part of the field, even in the presence of noise. Therefore, the choice of the observable is the determinant for parameter identification. (c) 2011 Optical Society of America

OCIS codes: $250.5960,140.1540,060.4785$.

Semiconductor laser (SL)-based systems with optical or electro-optical feedback have attracted a lot of attention in the chaos cryptography community due to their capability to develop broadband chaos within which gigabit messages can be encoded [1]. In this paradigm of communications, security relies mainly on the difficulty of identifying the emitter parameters necessary to build an adequate receiver that can synchronize with it [2]. To improve the security in such systems, it is required first to identify any eventual loopholes. In particular, it has been found that the delay time, while playing a main role in chaos generation, is, in many systems, vulnerable to identification from time series analysis [3-6]

In SL with all-optical feedback, it has been suggested that the time delay can be hidden when the parameters are such that it is close to the relaxation period of the laser operating with moderate feedback $[7,8]$. In this case, the interplay between the intrinsic SL dynamics and the delayed feedback leads to a concealment of the delay time. These results have been experimentally verified recently [9]. Both theoretical and experimental results have been obtained by computing statistical quantifiers from the intensity time series.

However, in principle, these results can depend on the observable [10]. Although being more difficult to measure, the phase of the electrical field is also part of the signal transmitted through the public channel; therefore, it can also be used by an eventual eavesdropper for data analysis. In this Letter we discuss the role of the phase in delay time identification. In particular, we show that for SL with optical feedback, even if the delay time is hidden in the intensity time series, it can be readily identified from the phase or from the quadrature time series.

We consider an SL with optical feedback operating at a regime for which the delay time is close to the relaxation oscillation time, as in [7]. The dynamics of the system can be described by the Lang-Kobayashi equations [11] for the slowly varying complex electric field amplitude $E(t)$ and the carrier number $N(t)$ inside the active layer:

$$
\frac{d E}{d t}=\frac{1+i \alpha}{2}\left(\mathcal{G}-\frac{1}{\tau_{p}}\right) E(t)+\kappa E_{T} e^{-i \omega_{0} T}+F(t),
$$

$$
\frac{d N}{d t}=J_{0}-\frac{N}{\tau_{e}}-\mathcal{G}|E|^{2},
$$

where the parameters are the photon lifetime $\tau_{p}$, carrier lifetime $\tau_{e}$, pump current $J_{0}$, feedback rate $\kappa$, linewidthenhancement factor $\alpha$, solitary laser frequency $\omega_{0}$, and delay time $T, E_{T} \equiv E(t-T)$. The gain is given by $\mathcal{G}=$ $g_{m}\left(N-N_{0}\right) /\left(1+\mathrm{Q}|E|^{2}\right)$ where $\mathrm{Q}$ is the saturation factor and $N_{0}$ is the carrier density at transparency. Spontaneous emission is modeled by $F(t)=\sqrt{2 \beta N(t)} \xi(t)$, where $\beta$ is the spontaneous emission rate and $\xi(t)$ is a complex Gaussian white noise with $\langle\xi(t)\rangle=0$ and $\left\langle\xi(t) \xi^{*}\left(t^{\prime}\right)\right\rangle=2 \delta\left(t-t^{\prime}\right)$. We consider [7]]: $\tau_{p}=2 \mathrm{ps}$, $\tau_{e}=2 \mathrm{~ns}, \alpha=5, \omega_{0} T=0, g_{m}=7.5 \times 10^{-\overline{13}} \mathrm{~m}^{3} \mathrm{~s}^{-1}, N_{0}=$ $3 \times 10^{24} \mathrm{~m}^{3}, \quad J_{\text {th }}=1.83 \times 10^{33} \mathrm{~m}^{-3} \mathrm{~s}^{-1}, J_{0}=1.05 J_{\text {th }}, \varrho=$ $2.5 \times 10^{-23} \mathrm{~m}^{3}$. With those parameters, the relaxation period is $\tau_{R 0}=0.75 \mathrm{~ns}$. Delay time concealment for moderate feedback rate was found for $T \in\left[\tau_{R 0}+\right.$ $\left.0.15 \mathrm{~ns}, \tau_{R 0}+0.35 \mathrm{~ns}\right]$ when analyzing $I(t) \equiv|E(t)|^{2}[7]$.

Statistical methods to identify the delay time include the autocorrelation function (AC), delayed mutual information (DMI), extrema statistics, and filling factor $[3,5-7]$. Out of those, $\mathrm{AC}$ and DMI are robust to noise and therefore are suitable to crack the time delay. For a time series $x(t)$, the $\mathrm{AC}$ is defined as

$$
C(s)=\frac{\left\langle[x(t)-\langle x(t)\rangle]\left[x_{s}(t)-\langle x(t)\rangle\right]\right\rangle}{\left\langle[x(t)-\langle x(t)\rangle]^{2}\right\rangle},
$$

where $x_{s}(t)=x(t-s)$. The DMI measures the information on $x(t)$ that can be obtained by observing $x_{s}(t)$

$$
D(s)=\sum_{x(t), x_{s}(t)} p\left(x(t), x_{s}(t)\right) \ln \frac{p\left(x(t), x_{s}(t)\right)}{p(x(t)) p\left(x_{s}(t)\right)},
$$

where $p(x(t))$ is the probability distribution function of $x(t)$ while $p\left(x(t), x_{s}(t)\right)$ is the joint probability distribution function.

Figure 1 displays the results for the $\mathrm{AC}$ and the DMI as computed from $I(t)$ for $\beta=0$. Here we only consider the cases for which the system is chaotic. For moderate values of the feedback rate, such as $\kappa=2.5 \mathrm{~ns}^{-1}$ 

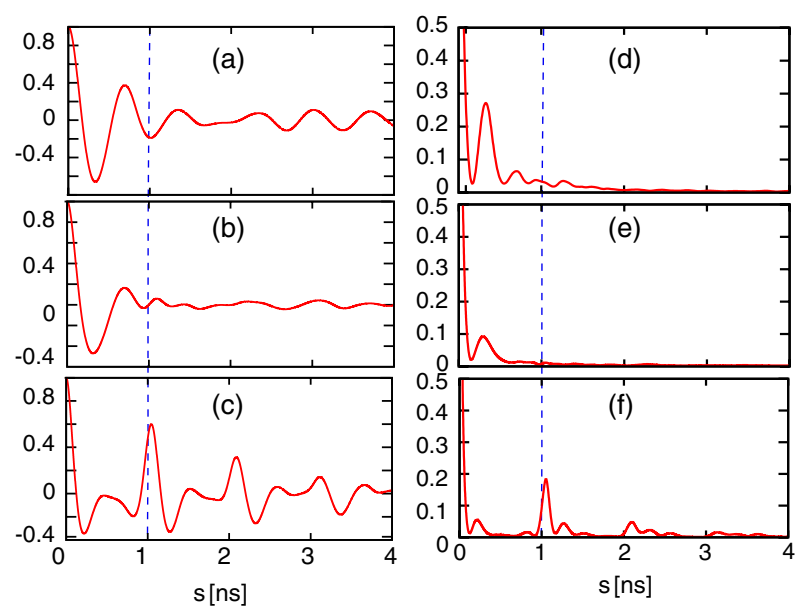

Fig. 1. (Color online) AC (left) and DMI (right) computed from $I(t)$ disregarding noise $(\beta=0)$ for $\kappa=2.5 \mathrm{~ns}^{-1}$, (a), (d) $\kappa=5 \mathrm{~ns}^{-1}$ (b), (e) and $\kappa=10 \mathrm{~ns}^{-1}$. (c), (f) Vertical dashes indicate the delay time $T=1 \mathrm{~ns}$. We have used a time series with $5 \times 10^{6}$ data points sampled every $10 \mathrm{ps}$.

[Figs. $1(\mathrm{a})$ and $\underline{1(\mathrm{~d})}$ ] and $\kappa=5 \mathrm{~ns}^{-1}$ [Figs. 1(b) and $\underline{1(\mathrm{e})]}$, the delay time (vertical dashed line) cannot be easily identified, even in the absence of noise. As shown in [7] the more pronounced maxima of the DMI and minima of the $\mathrm{AC}$ are located around $\tau_{R 0} / 2$, while there is no significant trace of the delay time. This is an effect of the interplay between the feedback and the intrinsic SL dynamics. For a larger feedback rate, the contribution of the delay term in Eq. (1) becomes more important, so that the delay time is no longer concealed. For example, for $\kappa=10 \mathrm{~ns}^{-1}$, a clear peak is observed around the delay time both in autocorrelation [Fig. 1(c)] and DMI [Fig. 1(f)] functions, as is also shown in $[\underline{7}, \overline{8}]$ for the same parameters.

While $I(t)$ can be measured by an eventual eavesdropper through direct detection (using a photodiode) of the transmitted signal, the phase, $\varphi(t) \equiv \operatorname{Arg}[E(t)]$, can also be detected using more sophisticated devices, such as an optical $90^{\circ}$ hybrid coupler $[12,13]$. The knowledge of $I(t)$ and $\varphi(t)$ allows the reconstruction of real and imaginary parts of the complex field.

Figure 2 displays the $\mathrm{AC}$ and the DMI computed from the phase recovered within the interval $[-\pi, \pi]$, and from
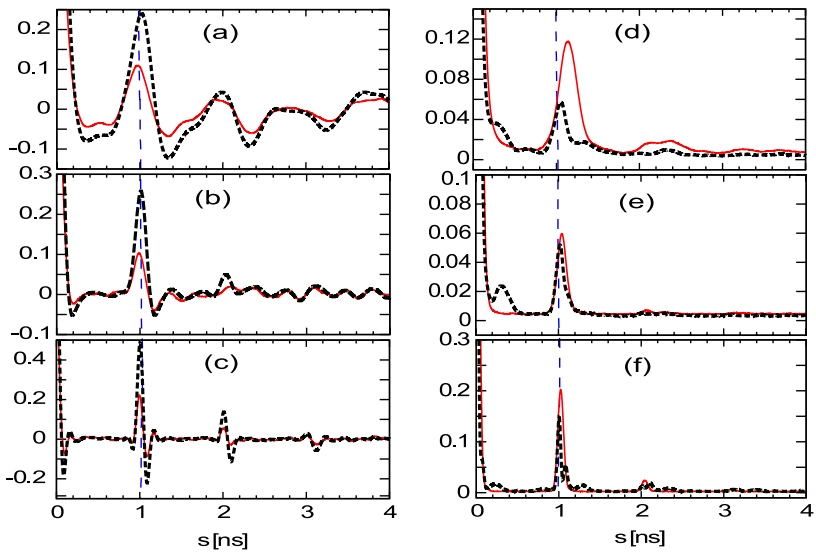

Fig. 2. (Color online) AC (left) and DMI (right), as in Fig. 1, but computed from $\varphi(t)$ (solid line) and $\mathcal{R} e[E(t)]$ (dotted line) and considering $\beta=10^{3} \mathrm{~s}^{-1}$. the real part $\mathcal{R} e[E(t)]$ of the electric field when spontaneous emission is included. Despite the presence of noise, the delay time can be identified even for $\kappa=$ $2.5 \mathrm{~ns}^{-1}$ in both quantifiers, although the DMI peak is narrower and thus more distinguishable. The peak for the DMI appears at a slightly larger value as compared to the actual value of the delay time, while the peak for the $\mathrm{AC}$ is located very close to the actual delay time. As the feedback rate increases, both the AC and DMI peaks become narrower and more distinguishable, while the location of the DMI peak gets closer to the actual delay time. Finally, for $\kappa=10 \mathrm{~ns}^{-1}$, for which the delay signature was already found from the intensity series, the peaks computed from $\varphi(t)$ or $\mathcal{R} e[E(t)]$ are sharper and more precisely located than those obtained from $I(t)$. For the imaginary part of the field or for other quadratures, one obtains similar results to those obtained for the real part.

Globally, the peak for the phase in the DMI is larger than that obtained from the real part of the field, while the opposite occurs for the AC. This indicates that the phase indeed carries a nonlinear relationship between its state and its lagged version. Linear relationships, such as the ones measured by the correlation, turn out to be stronger for the quadratures.

The different results for intensity and phase can be explained from the dynamics. From Eq. (1), neglecting noise, we have

$$
\frac{d \varphi}{d t}=\frac{\alpha}{2}\left(\mathcal{G}-\frac{1}{\tau_{p}}\right)+\kappa \frac{\left|E_{T}\right|}{|E|} \sin \left(\varphi_{T}-\varphi-\omega_{0} T\right) .
$$

The last term tends to keep constant the difference $\varphi_{T}-\varphi$, so that the dynamics of $\varphi_{T}$ is strongly linked to that of $\varphi$. Contrarily, the equation for the intensity does not have a term depending on $I-I_{T}$ that could push $I$ close to $I_{T}$.

Figure 3 (left column) shows the size of the peak found in the DMII computed from the intensity, phase, and real part of the field as a function of the feedback strength for $T=1 \mathrm{~ns}$. As an indication of the background level, we
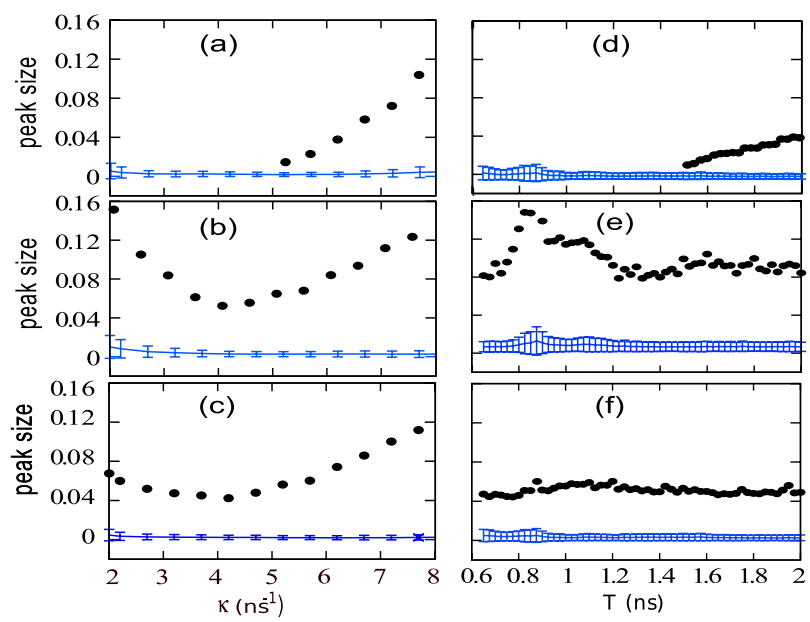

Fig. 3. (Color online) Size of the DMI peak signaling the delay computed from (a), (d) $I(t)$, (b), (e) $\varphi(t)$, and (c), (f) $\mathcal{R} e[E(t)]$, considering $\beta=10^{3} \mathrm{~s}^{-1}$. For the left column, $T=1 \mathrm{~ns}$, while for the right column, $\kappa=2.5 \mathrm{~ns}^{-1}$. Solid lines and bars indicate the background mean value and standard deviation. 

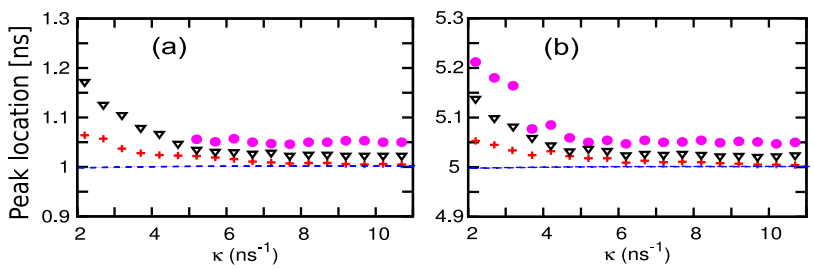

Fig. 4. (Color online) DMI peak location computed from $I(t)$ $(\bullet), \varphi(t)(\boldsymbol{\nabla})$ and $\mathcal{R e}(E(t))(+)$ for (a) $T=1$ ns and (b) $5 \mathrm{~ns}$. (b) Horizontal dashed lines indicate the delay time. $\beta=0$.

also show the average and standard deviation (solid line with bars) of the DMI maxima on the interval from $T+$ $0.5 \mathrm{~ns}$ to $T+2.5 \mathrm{~ns}$. Two main regions can be identified: for $\kappa \gtrsim 6 \mathrm{~ns}^{-1}$, it is possible to distinguish a peak around the delay time from the background level independently to the variable used. Furthermore, in this region the peak size increases linearly with the feedback strength. For smaller values of $\kappa$, the peak size depends strongly on the chosen variable. While the peak obtained from the intensity merges with the background, the one from the phase or the real part remains always distinguishable. Figure 3 (right column) shows the size of the DMI peak as a function of $T$. For $I(t)$, the peak practically vanishes for $T \lesssim 1.5 \mathrm{~ns}$ while it remains clearly visible for $\varphi(t)$ or $\mathcal{R} e[E(t)]$ in the whole range explored. For $\varphi(t)$, the size of the peak is in fact larger for $T \approx \tau_{R 0}$, while for $\mathcal{R} e[E(t)]$, it is practically independent of $T$.

Figure 4 compares the location of the DMI peaks computed from different variables, neglecting noise. $\mathcal{R} e[E(t)]$ appears as the variable from which the delay time can be retrieved more accurately, even if it is close to the relaxation period. The peak for the $I(t)$ is not only the less visible, it is also the one located further away from the actual delay time. This is true for $T=1 \mathrm{~ns}$ and also for longer delays, such as $T=5 \mathrm{~ns}$. Similar results are obtained when noise is present.

In summary, we have shown that in SL with external feedback, the delay time can be identified from the phase or quadrature time series by the means of AC or DMI, even when it is concealed in the intensity time series. Besides providing more visible peaks, phase and quadratures also lead to a more precise location of the peak. The difference in the information that can be extracted by analyzing intensity and phase can be further illustrated by looking at the power and optical spectra. While the power spectrum computed from the noiseless intensity does not reveal the presence of a delay time [Fig. 5(a)], the optical spectrum, which depends on both intensity and phase, does, even in a noisy system: the delay time defines the spacing between the modes around the maximum [Fig. 5(b)]. In SL, the $\alpha$-factor determines the strength of the coupling between amplitude and phase dynamics. By increasing $\alpha$, this coupling becomes stronger, and thus the size of the DMI or autocorrelation peaks computed from the phase decrease. However, we
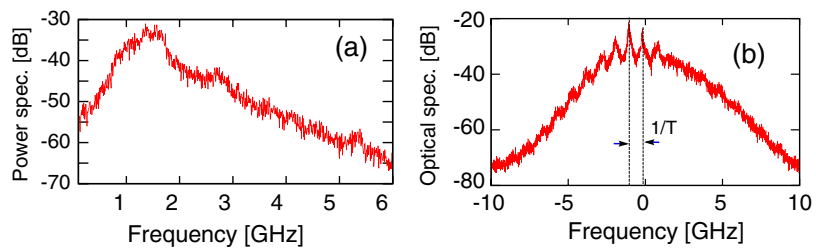

Fig. 5. (Color online) (a) Power and (b) optical spectra for $\kappa=2.5 \mathrm{~ns}^{-1}, T=1 \mathrm{~ns}$ and for (a) $\beta=0$ and (b) $\beta=10^{3} \mathrm{~s}^{-1}$.

have checked that even for $\alpha=10$, the DMI peaks for phase and quadratures are still distinguishable. As a final remark, we note that in systems with electro-optical feedback, the time delay can indeed be concealed when analyzing the phase; however, this concealment requires more sophisticated schemes [14]].

Financial support from the Ministerio de Ciencia e Innovacion (MICINN), Spain, and the Fondo Europeo de Desarrollo Regional under projects FIS2007-60327 [Física Interdisciplinar de Sistemas Complejos (FISICOS)] and TEC2009-14101 [Delay-Coupled Diode Lasers for Photonic Applications (DeCoDicA)] and by the European Commission (EC) Project PHOCUS (FP7-ICT-2009-C240763) is acknowledged. R. M. Nguimdo also acknowledges fellowship BES-2007-14627 under the FPI program of MICINN.

\section{References}

1. A. Argyris, D. Syvridis, L. Larger, V. Annovazzi-Lodi, P. Colet, I. Fischer, J. García-Ojalvo, C. R. Mirasso, L. Pesquera, and K. A. Shore, Nature 438, 343 (2005).

2. X. Li, W. Pan, B. Luo, and D. Ma, IEEE J. Quantum Electron. 42, 953 (2006).

3. R. Hegger, M. J. Bünner, H. Kantz, and A. Giaquinta, Phys. Rev. Lett. 81, 558 (1998).

4. V. S. Udaltsov, J. P. Goedgebuer, L. Larger, J. B. Cuenot, P. Levy, and W. T. Rhodes, Phys. Lett. A 308, 54 (2003).

5. M. D. Prokhorov, V. I. Ponomarenko, A. S. Karavaev, and B. P. Bezruchko, Phys. D 203, 209 (2005).

6. L. Zunino, M. C. Soriano, I. Fischer, O. A. Rosso, and C. R. Mirasso, Phys. Rev. E 82, 046212 (2010).

7. D. Rontani, A. Locquet, M. Sciamanna, and D. S. Citrin, Opt. Lett. 32, 2960 (2007).

8. D. Rontani, A. Locquet, M. Sciamanna, D. S. Citrin, and S. Ortín, IEEE J. Quantum Electron. 45, 879 (2009).

9. J. G. Wu, G. Q. Xia, X. Tang, X. D. Lin, T. Deng, L. Fan, and Z. M. Wu, Opt. Express 18, 6661 (2010).

10. C. Letellier, L. A. Aguirre, and J. Maquet, Phys. Rev. E 71, 066213 (2005).

11. R. Lang and K. Kobayashi, IEEE J. Quantum Electron. 16, 347 (1980).

12. H. C. Wang, K. P. Ho, H. K. Chen, and H. C. Lu, J. Lightwave Technol. 24, 5075 (2006).

13. L. Zimmermann, K. Voigt, G. Winzer, K. Petermann, and C. M. Weinert, IEEE Photon. Technol. Lett. 21, 143 (2009).

14. R. M. Nguimdo, P. Colet, L. Larger, and L. Pesquera, Phys. Rev. Lett. 107, 034103 (2011). 